\title{
Reduced-Complexity Multicell Decoding Systems with Multiple Antennas at the Base Station
}

\author{
Symeon Chatzinotas, Muhammad Ali Imran, Reza Hoshyar \\ Centre for Communication Systems Research, University of Surrey, United Kingdom, GU2 7XH \\ \{S.Chatzinotas, M.Imran, R.Hoshyar\}@surrey.ac.uk
}

\begin{abstract}
Multicell joint decoding has been proven to greatly enhance the capacity of cellular systems in a range of regimes. However, the complexity of such a joint receiver makes it impossible to implement in practice using current computational capabilities. In this direction, this paper investigates the capacity performance of reduced-complexity communication schemes in order to evaluate their performance with respect to the optimal multicell joint decoding scheme. More specifically, two sub-optimal schemes are considered: 1) intracell user orthogonalization combined with optimal multicell joint decoding and 2) intra-cell user orthogonalization combined with linear MMSE filtering and single-user decoding. The employed cellular multiple-access channel model incorporates flat fading, path loss, distributed users and multiple antennas at the Base Station, while both peak and average transmit power constraints are taken into account. In this context, it is shown that linear MMSE filtering combined with multiple BS antennas and intra-cell orthogonalization can still provide a considerable capacity enhancement. Furthermore, FDMA is shown to be more efficient than TDMA as an intra-cell orthogonalization technique.
\end{abstract}

\section{Categories and Subject Descriptors}

C.2.1 [Computer-communication Networks]: Network Architecture and Design-Wireless communication; E.4 [Data]: Coding and Information Theory-Formal models of communication

\section{General Terms}

Theory, Performance

\section{Keywords}

Multicell Decoding, Multi-antenna Systems, Sum-rate Capacity, MMSE filtering

\section{INTRODUCTION}

Permission to make digital or hard copies of all or part of this work for personal or classroom use is granted without fee provided that copies are not made or distributed for profit or commercial advantage and that copies bear this notice and the full citation on the first page. To copy otherwise, to republish, to post on servers or to redistribute to lists, requires prior specific permission and/or a fee.

IWCMC '09, June 21-24, 2009, Leipzig, Germany

Copyright 2009 ACM 978-1-60558-569-7/09/06 ...\$5.00.
Since the seminal work of Wyner [1], Base Station (BS) cooperation and multicell decoding have been studied extensively in a wide range of regimes. According to this paradigm, the BSs are interconnected through broadband error-free links to a central processor (hyper-receiver), which jointly decodes the received signals. The hyper-receiver is assumed to have perfect channel knowledge and infinite processing capabilities. Multicell decoding models have gradually evolved by incorporating more realistic characteristics of the wireless cellular channel, such as fading and path loss $[2,3,4]$, User Terminal (UT) distribution [5], clustering [6] and multiple antennas [7]. In all the aforementioned studies, it was established that multicell decoding effectively eliminates the interference-limited behavior of the per-cell capacity. Furthermore, in order to achieve the optimal capacity in a cellular multiple-access channel, all the UTs have to transmit simultaneously over the ensemble of the channel timefrequency resources, while Successive Interference Cancellation (SIC) is utilized at the joint processor [2] to recover the individual user signals. However, the complexity of such a receiver grows exponentially with the number of UTs which are involved in the SIC process [8] and in addition, successive decoding techniques can introduce error propagation in the decoding process.

In this direction, this paper investigates the capacity performance of reduced-complexity communication schemes in order to evaluate their performance with respect to the optimal multicell joint decoding scheme. The complexity can be reduced either by decreasing the number of UTs in the SIC process or by using sub-optimal multiuser receivers, which can still exploit the paradigm of BS cooperation. More specifically, the number of UTs can be decreased by splitting the intra-cell UTs into orthogonal groups using TDMA or FDMA techniques $[2,4,3]$. Additionally, the computationally expensive multicell joint decoder could be replaced by the simpler approach of linear Minimum Mean Square Error (MMSE) filtering, followed by single-UT decoding [8]. This receiver is characterized by linear complexity in the number of users, or at least polynomial if one considers that the computation of the MMSE filters, matrix-vector multiplications and subtraction are quadratic or cubic in the number of users [8]. In this direction, we consider two sub-optimal schemes: 1) intra-cell user orthogonalization combined with multicell joint decoding and 2) intra-cell user orthogonalization combined with linear MMSE filtering and single-user decoding. Furthermore, we introduce a comprehensive cellular multiple-access channel model, which includes flat fad- 


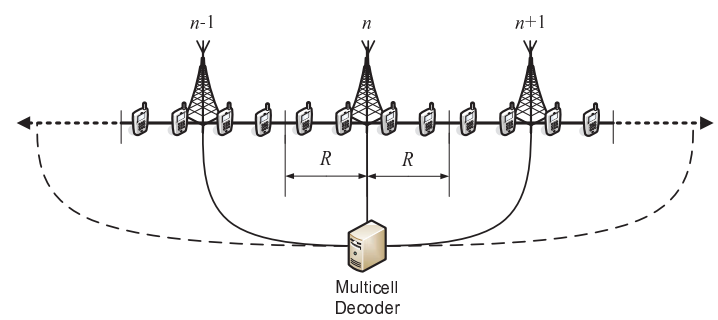

Figure 1: Linear cellular array with $K=4$ UTs per cell on a uniform grid.

ing, path loss, distributed UTs and multiple antennas at the Base Station. A relevant investigation can be found in [4], although therein a single multiple-antenna UT per cell is considered in combination with single-cell linear MMSE detectors or nonlinear MMSE SIC detectors. In our investigation, multiple UTs per cell with arbitrary number of antennas per UT can transmit simultaneously, as long as the total number of transmit antennas per cell matches the number of receive antennas at the BS. Furthermore, a single linear MMSE filter is applied across all the cooperating cells, which aims at jointly maximizing the achieved Signal to Interference and Noise Ratio (SINR). In this context, TDMA and FDMA are utilized as intra-cell orthogonalization techniques, while both peak and average transmit power constraints are taken into account.

\section{CHANNEL MODEL}

Assume that $K$ UTs are spatially distributed in each cell (with radius $R$ as in Figure 1) of a linear cellular array comprising $N$ BSs with $n_{B S}$ antennas per BS. Furthermore, we assume that Channel State Information (CSI) is not available at the UT-side and thus input optimization through iterative waterfilling is not possible. In the remainder of the analysis we consider single-antenna UTs to simplify the notations, although the model is straightforwardly extendable to UTs equipped with arbitrary number of antennas. It should also be noted that perfect CSI is assumed to be available at the receiver-side. In this context and considering an average power constraint $\bar{P}$ per UT antenna, the optimal transmission strategy is non-orthogonal transmission with power $\bar{P}$ followed by optimal multicell joint decoding [2]. In other words, the UTs transmit simultaneously on the available bandwidth and SIC is employed at the central multicell-decoding processor. Furhtermore, we consider a receiver which performs linear MMSE filtering followed by single-user decoding. The main limitation of the MMSE receiver is that the number of UTs that can be filtered effectively is limited by the rank of the channel matrix, namely the number of receive antennas $N n_{B S}$. Due to this factor, intra-cell orthogonalization techniques have to applied amongst the system UTs, allowing only $N n_{B S}$ out of the $K N$ UTs to transmit over the same channel resources. In this direction, TDMA and FDMA are considered as means of dividing the system UTs into $K / n_{B S}$ orthogonal groups. In addition, we introduce a further practical consideration, namely a peak power constraint $P_{\max }$, by defining the Peak to Average power Ratio $\mathrm{PAR}=P_{\max } / \bar{P}$ with $\mathrm{PAR} \geq 1$. In practical cellular systems, the concept of peak power constraint can be justified based on the non-linearity of ampli- fiers, air-interface standardization, interference regulations and health regulations.

\subsection{Optimal Transmission Strategy}

Let us assume a large number of uniformly distributed UTs and a channel model impaired by flat fading and path loss. Adopting the optimal transmission strategy, the received signal at $\mathrm{BS} n$, at time index $i$, will be given by the $n_{B S} \times 1$ vector:

$$
\mathbf{y}^{n}[i]=\sum_{j=1}^{N} \sum_{k=1}^{K} \varsigma_{k}^{j n} \mathbf{g}_{k}^{j n}[i] x_{k}^{j}[i]+\mathbf{z}^{n}[i],
$$

where $x_{k}^{j}[i]$ is the $i$ th complex channel symbol transmitted by the $k$ th UT of the $j$ th cell and $\mathbf{g}_{k}^{j n}$ is a $n_{B S} \times$ 1 Gaussian vector containing independent identically distributed (i.i.d.) complex circularly symmetric (c.c.s.) elements of unit variance, which represent the flat fading processes experienced in the transmission path between the $n_{B S}$ antennas of the $n$th $\mathrm{BS}$ and the $k$ th UT in the $j$ th cell. The fading coefficients are assumed to have unit power, i.e. $\mathbb{E}\left[\mathbf{g}_{k}^{j n}[i]\left(\mathbf{g}_{k}^{j n}[i]\right)^{\dagger}\right]=\mathbf{I}_{n_{B S}}$ for all $(n, j, k)$. The path-loss coefficients $\varsigma_{k}^{j n}$ in the transmission path between the $n$th BS and the $k$ th $\mathrm{UT}$ in the $j$ th cell are calculated according to the power-law path loss model $\varsigma_{k}^{j n}=\left(1+d_{k}^{j n}\right)^{-\eta / 2}$, where $d_{k}^{j n}$ is the distance between the $n$th BS and the $k$ th UT in the $j$ th cell and $\eta$ is the path-loss exponent. The $n_{B S} \times 1$ vector $\mathbf{z}^{n}=\left[\begin{array}{lll}z^{1} & \ldots & z^{n_{B S}}\end{array}\right]^{T}$ contains i.i.d. c.c.s. random variables representing Additive White Gaussian Noise (AWGN) with $\mathbb{E}\left[\mathbf{z}^{n}\right]=\mathbf{0}, \mathbb{E}\left[\mathbf{z}^{n}\left(\mathbf{z}^{n}\right)^{\dagger}\right]=\sigma^{2} \mathbf{I}$. Since there is no CSI at the UT-side and PAR $\geq 1$, all UTs transmit with a constant power $P$, i.e. $\left|x_{k}^{j}[i]\right|^{2}=P$ for all $(k, j, i)$, assuming a flat fading environment. The model can be more compactly expressed as a vector memoryless channel of the form $\mathbf{y}=\mathbf{H x}+\mathbf{z}$, where $\mathbf{y}=\left[\overline{\mathbf{y}}^{1} \ldots \overline{\mathbf{y}}^{N}\right]^{T}$ with $\overline{\mathbf{y}}^{n}=\left(\mathbf{y}^{n}\right)^{T}$ (c.f. Eq.(1)) represents received signals by the multiple antennas of all the $\mathrm{BSs}, \mathbf{x}=\left[\begin{array}{lllll}x_{1}^{1} \ldots x_{K}^{1} x_{1}^{2} \ldots & \ldots & x_{K}^{N-1} x_{1}^{N} \ldots x_{K}^{N}\end{array}\right]^{T}$ represents transmit signals by all the UTs of the cellular system and $\mathbf{z}=\left[\overline{\mathbf{z}}^{1} \ldots \overline{\mathbf{z}}^{N}\right]^{T}$ with $\overline{\mathbf{z}}^{n}=\left(\mathbf{z}^{n}\right)^{T}$ (c.f. Eq.(1)) represents AWGN. The rectangular channel matrix $\mathbf{H}$ can be written as $\mathbf{H}=\left(\boldsymbol{\Sigma} \otimes \mathbf{1}_{n_{B S}}\right) \odot \mathbf{G}$, where $\boldsymbol{\Sigma}$ is a $N \times K N$ deterministic matrix, $\mathbf{1}_{n_{B S}}$ is a $n_{B S} \times 1$ vector of ones, and $\mathbf{G} \sim \mathcal{C N}\left(\mathbf{0}, \mathbf{I}_{N n_{B S}}\right)$ is a complex Gaussian $N n_{B S} \times K N$ matrix comprising the corresponding Rayleigh fading coefficients. Furthermore, $\otimes$ and $\odot$ denote the Kronecker and Hadamard (element-wise) matrix product respectively. The entries of the $\boldsymbol{\Sigma}$ matrix are defined by the variance profile function $\varsigma(u, v)=(1+d(u, v))^{-\eta / 2}$, where $u \in(0,1]$ and $v \in(0, K]$ are the normalized indices for the BSs and the UTs respectively and $d(u, v)$ denotes the distance between BS $u$ and user $v$.

\subsection{Intra-cell Orthogonalization}

Considering intra-cell orthogonalization, only $N n_{B S}$ out of $K N$ UTs transmit per channel use and thus the received signal at $\mathrm{BS} n$, at time index $i$, will be given by:

$$
\mathbf{y}^{n}[i]=\sum_{j=1}^{N} \sum_{k=1}^{n_{B S}} \varsigma_{k}^{j n} \mathbf{g}_{k}^{j n}[i] x_{k}^{j}[i]+\mathbf{z}^{n}[i],
$$

or expressed as a vector memoryless channel of the form $\mathbf{y}=$ $\mathbf{H}_{\mathbf{o}} \mathbf{x}_{\mathbf{o}}+\mathbf{z}$, where $\mathbf{x}_{\mathbf{o}}=\left[x_{1}^{1} \ldots x_{n_{B S}}^{1} x_{1}^{2} \ldots \ldots x_{n_{B S}}^{N-1} x_{1}^{N} \ldots x_{n_{B S}}^{N}\right]^{T}$ represents transmit signals by a group of $N n_{B S}$ UTs. The 
square channel matrix $\mathbf{H}_{\mathbf{o}}$ can be written as $\mathbf{H}_{\mathbf{o}}=\left(\boldsymbol{\Sigma}_{\mathbf{o}} \otimes \mathbf{1}_{n_{B S}}\right) \odot$ $\mathbf{G}_{\mathbf{o}}$, where $\boldsymbol{\Sigma}_{\mathbf{o}}$ is a $N \times N n_{B S}$ deterministic matrix, $\mathbf{1}_{n_{B S}}$ is a $n_{B S} \times 1$ vector of ones, and $\mathbf{G}_{\mathbf{o}} \sim \mathcal{C N}\left(\mathbf{0}, \mathbf{I}_{N n_{B S}}\right)$ is a complex Gaussian $N n_{B S} \times N n_{B S}$ matrix comprising the corresponding Rayleigh fading coefficients. The entries of the $\boldsymbol{\Sigma}_{\mathbf{o}} \mathrm{O}$ matrix are still defined by the variance profile function $\varsigma(u, v)=(1+d(u, v))^{-\eta / 2}$ with the differentiation that $u \in(0,1]$ and $v \in\left(0, n_{B S}\right]$.

\section{CAPACITY ANALYSIS}

\subsection{Optimal Joint Decoding}

Considering the optimal transmission strategy combined with optimal multicell joint decoding, the asymptotic percell sum-rate capacity $C_{\text {opt }}$ for this model is given by [9]

$$
\begin{aligned}
& C_{\text {opt }}=\lim _{N \rightarrow \infty} \frac{1}{N} \mathbb{E}[\mathcal{I}(\mathbf{x} ; \mathbf{y} \mid \mathbf{H})] \\
& =\lim _{N \rightarrow \infty} \frac{1}{N} \mathbb{E}\left[\log \operatorname{det}\left(\mathbf{I}_{N}+\bar{\gamma} \mathbf{H H}^{\dagger}\right)\right] \\
& =\lim _{N \rightarrow \infty} n_{B S} \mathbb{E}\left[\frac{1}{N n_{B S}} \sum_{i=1}^{N n_{B S}} \log \left(1+\frac{\hat{\gamma}}{K} \lambda_{i}\left(\frac{1}{N} \mathbf{H H}^{\dagger}\right)\right)\right] \\
& =n_{B S} \int_{0}^{\infty} \log \left(1+\frac{\hat{\gamma}}{K} x\right) d \mathrm{~F}_{\frac{1}{N} \mathbf{H H}^{\dagger}}(x) \\
& =n_{B S} \mathcal{V}_{\frac{1}{N} \mathbf{H H}^{\dagger}}(\hat{\gamma} / K)=K \mathcal{V}_{\frac{1}{N} \mathbf{H}^{\dagger} \mathbf{H}(\hat{\gamma} / K),}
\end{aligned}
$$

where $\hat{\gamma}=K N \bar{\gamma}$ and $\bar{\gamma}=\bar{P} / \sigma^{2}$ is the system- and UTtransmit power normalized with the receiver noise power respectively, $\lambda_{i}(\mathbf{X})$ are the eigenvalues of matrix $\mathbf{X}$ and $\mathcal{V}_{\mathbf{X}}$ is the Shannon transform [9] of a random square Hermitian matrix $\mathbf{X}$. For $\mathbf{G} \sim \mathcal{C} \mathcal{N}\left(\mathbf{0}, \mathbf{I}_{N}\right)$, the empirical eigenvalue distribution of $\frac{1}{N} \mathbf{G}^{\dagger} \mathbf{G}$ converges almost surely (a.s.) to the nonrandom limiting eigenvalue distribution of the Marcenko-Pastur law, whose Shannon transform with parameter $\delta$ is given by $\mathcal{V}_{\frac{1}{N} \mathbf{G}^{\dagger} \mathbf{G}}(\delta) \stackrel{a . s .}{\longrightarrow} \mathcal{V}_{\mathrm{MP}}(\delta, \beta)$ where $\beta$ is the ratio of the horizontal to the vertical dimension of the $\mathbf{G}$ matrix [9, Example 2.14]. According to the Free Probability approach in $[3,5]$, if $\boldsymbol{\Sigma}$ is a path loss dependent $N \times K N$ deterministic matrix, the limiting eigenvalue distribution of $(1 / N) \mathbf{H}^{\dagger} \mathbf{H}$ and its Shannon transform is approximated by a scaled version of the Marcenko-Pastur law

$$
\mathcal{V}_{\frac{1}{N} \mathbf{H}^{\dagger} \mathbf{H}}(\hat{\gamma} / K) \simeq \mathcal{V}_{\mathrm{MP}}\left(q(\boldsymbol{\Sigma}) \hat{\gamma} / K, K / n_{B S}\right)
$$

where $q(\boldsymbol{\Sigma}) \triangleq\|\boldsymbol{\Sigma}\|^{2} / K N^{2}$ with $\|\boldsymbol{\Sigma}\| \triangleq \sqrt{\operatorname{tr}\left\{\boldsymbol{\Sigma}^{\dagger} \boldsymbol{\Sigma}\right\}}$ being the Frobenius norm of the $\boldsymbol{\Sigma}$ matrix. In the asymptotic case $q(\boldsymbol{\Sigma})$ is given by

$$
\lim _{N \rightarrow \infty} q(\boldsymbol{\Sigma})=\frac{1}{K} \int_{0}^{1} \int_{0}^{K} \varsigma^{2}(u, v) d u d v .
$$

Since the variance profile function defines a rectangular blockcirculant matrix with $1 \times K$ blocks which is symmetric about $v=K u$, the matrix $\boldsymbol{\Sigma}$ is asymptotically row-regular [9, Def. 2.10] and thus the previous equation can be simplified to

$$
\lim _{N \rightarrow \infty} q(\boldsymbol{\Sigma})=\frac{1}{K} \int_{0}^{K} \varsigma^{2}(v) d v, \forall u \in(0,1] .
$$

In conclusion, the optimal per-cell sum-rate capacity can be written as:

$$
C_{\mathrm{opt}}=K \mathcal{V}_{\mathrm{MP}}\left(q(\boldsymbol{\Sigma}) \hat{\gamma} / K, K / n_{B S}\right) .
$$

If optimal joint decoding is combined with intracell orthogonalization, then following a similar derivation the per-cell sum-rate capacity can be written as:

$$
C_{\mathrm{opt}}^{\mathrm{orth}}=n_{B S} \mathcal{V}_{\mathrm{MP}}\left(q\left(\boldsymbol{\Sigma}_{\mathbf{o}}\right) \tilde{\gamma} / n_{B S}, 1\right),
$$

where $\tilde{\gamma}=N n_{B S} \gamma$ and $\gamma$ is the group- and UT- transmit power normalized with the receiver noise power respectively. We do not use $\bar{\gamma}$ for the normalized UT transmit power, since its value depends on the orthogonalization technique and the PAR. This dependence is described in detail later on in this section. Now let us assume that the number of BS antennas $n_{B S}$ is sufficiently large, so that even if $K$ UTs are divided in orthogonal groups of $n_{B S}$ UTs, there is still a large number of UTs per group uniformly distributed across the coverage area of each cell. In this case, it can be written that

$$
\lim _{N \rightarrow \infty} q\left(\boldsymbol{\Sigma}_{\mathbf{o}}\right)=\frac{1}{n_{B S}} \int_{0}^{n_{B S}} \varsigma^{2}(v) d v .
$$

\subsection{Linear MMSE Receiver}

Let us consider the suboptimal scenario, where intra-cell orthogonalization is employed as the transmission strategy, followed by linear MMSE filtering and single-UT decoding. If SINR avg $_{\text {and }}$ mmse $_{\text {avg }}$ denote the average UT SINR and MMSE after filtering, the achieved per-cell capacity can be approximated by [9, Equation 1.9][8, 10, 11]:

$$
\begin{aligned}
& C_{\text {mmse }}=n_{B S} \lim _{N \rightarrow \infty} \log \left(1+\text { SINR }_{\text {avg }}\right) \\
& =n_{B S} \lim _{N \rightarrow \infty} \log \left(\text { mmse }_{\text {avg }}{ }^{-1}\right) \\
& =-n_{B S} \lim _{N \rightarrow \infty} \log \left(\frac{1}{N n_{B S}} \mathbb{E}\left[\operatorname{tr}\left\{\left(\mathbf{I}_{N n_{B S}}+\gamma \mathbf{H}_{\mathbf{o}}{ }^{\dagger} \mathbf{H}_{\mathbf{o}}\right)^{-1}\right\}\right]\right) \\
& =-n_{B S} \lim _{N \rightarrow \infty} \log \left(\mathbb{E}\left[\frac{1}{N n_{B S}} \sum_{j=1}^{N n_{B S}} \frac{1}{1+\frac{\tilde{\gamma}}{n_{B S}} \lambda_{j}\left(\frac{1}{N} \mathbf{H}_{\mathbf{o}}^{\dagger} \mathbf{H}_{\mathbf{o}}\right)}\right]\right) \\
& =-n_{B S} \log \left(\int_{0}^{\infty} \frac{1}{1+\frac{\tilde{\gamma}}{n_{B S}} x} f_{\frac{1}{N} \mathbf{H}_{\mathbf{o}}^{\dagger} \mathbf{H}_{\mathbf{o}}}^{\infty}(x) d x\right) \\
& =-n_{B S} \log \left(\eta_{\frac{1}{N} \mathbf{H}_{\mathbf{o}}^{\dagger} \mathbf{H}_{\mathbf{o}}}\left(\frac{\tilde{\gamma}}{n_{B S}}\right)\right) \text {, }
\end{aligned}
$$

where $\tilde{\gamma}=N n_{B S} \gamma$ and $\gamma$ is the group- and UT- transmit power normalized with the receiver noise power respectively and $\eta_{\mathbf{X}}$ is the $\eta$-transform [9] of a random square Hermitian matrix $\mathbf{X}$. For $\mathbf{G} \sim \mathcal{C N}\left(\mathbf{0}, \mathbf{I}_{N}\right)$, the $\eta$-transform of the Marcenko-Pastur $\eta_{\mathrm{MP}}(\delta, \beta)$ law is given in [9, Example 2.10]. According to the Free Probability approach in $[3,5]$, the $\eta$ transform of $(1 / N) \mathbf{H}_{\mathbf{o}}{ }^{\dagger} \mathbf{H}_{\mathbf{o}}$ can be written as:

$$
\eta_{\frac{1}{N} \mathbf{H}_{\mathbf{o}}^{\dagger} \mathbf{H}_{\mathbf{o}}}\left(\frac{\tilde{\gamma}}{n_{B S}}\right) \simeq \eta_{\mathrm{MP}}\left(q\left(\boldsymbol{\Sigma}_{\mathbf{o}}\right) \frac{\tilde{\gamma}}{n_{B S}}, 1\right)
$$

and the MMSE capacity

$$
C_{\mathrm{mmse}}=-n_{B S} \log \left(\eta_{\mathrm{MP}}\left(q\left(\boldsymbol{\Sigma}_{\mathbf{o}}\right) \frac{\tilde{\gamma}}{n_{B S}}, 1\right)\right) .
$$

\subsection{TDMA versus FDMA}

So far the treatment of intra-cell orthogonalization was common for both TDMA and FDMA. However, there are a number of distinct differences, which should be pointed out before we proceed to the numerical results section. Firstly, the assumption of flat fading is more valid for FDMA than 
Table 1: Practical parameters

\begin{tabular}{c|c|l} 
Parameter & Symbol & Value/Range \\
\hline Cell Radius & $R$ & $0.1-3 \mathrm{Km}$ \\
Reference Distance & $d_{0}$ & $1 \mathrm{~m}$ \\
Path Loss at ref. distance & $L_{0}$ & $34.5 \mathrm{~dB}$ \\
Path Loss Exponent & $\eta$ & 3.5 \\
UTs density & $K_{0}$ & $40(\mathrm{UTs} / \mathrm{Km})$ \\
UT Transmit Power & $P_{T}$ & $200 \mathrm{~mW}$ \\
Thermal Noise Density & $N_{0}$ & $-169 \mathrm{dBm} / \mathrm{Hz}$ \\
Channel Bandwidth & $B$ & $5 \mathrm{MHz}$ \\
\hline
\end{tabular}

TDMA systems due to the narrower subcarrier bands. Secondly, the capacity-achieving Gaussian input presumes codewords, which are sufficiently long to experience all possible realizations of the channel. Considering that each orthogonal UT group is scheduled once every $K / n_{B S}$ channel uses, there can be a considerable time delay between transmissions, which is problematic for delay-sensitive applications. Thirdly and most importantly, the TDMA transmit power can get saturated due to the peak power constraint. More specifically, as the number of orthogonal groups increases, the transmission duration of each group decreases and thus the UTs have to employ bursty transmission, namely short duration and high power. However, the peak power constraint of each UT limits the maximum instantaneous power which can be utilized and thus the TDMA transmit power gets saturated. This problem does not apply in FDMA systems, since in this case the SNR gain originates in the fact that narrower subcarrier bands are affected by lower noise levels. To quantify this distinctive difference, the TDMA transmit SNR can be written as

$$
\gamma=\gamma_{\mathrm{T}}= \begin{cases}\frac{\bar{P}}{\sigma^{2}} \cdot \frac{K}{n_{B S}} & \text { PAR } \geq \frac{K}{n_{B S}} \\ \frac{\bar{P}}{\sigma^{2}} \cdot \mathrm{PAR} & \mathrm{PAR}<\frac{K}{n_{B S}}\end{cases}
$$

while the transmit FDMA SNR can be written as

$$
\gamma=\gamma_{\mathrm{F}}=\frac{\bar{P}}{\sigma^{2}} \cdot \frac{K}{n_{B S}} .
$$

\section{NUMERICAL RESULTS}

In order to produce meaningful results, the typical parameters of Table 1 will be considered for a real-world macrocellular scenario. In this context, assuming that the power loss at the reference distance $d_{0}$ is $L_{0}$, the scaled variance profile function is given by $\varsigma(d)=\sqrt{L_{0}\left(1+d / d_{0}\right)^{-\eta}}$, where $d$ is the distance in meters between the considered UT and BS. The values of $L_{0}$ and $\eta$ have been fitted to the path loss model defined in the "Urban Macro" scenario of [12].

\subsection{Capacity Simulations}

In each figure of this section, there are five curves: 1) optimal per-cell capacity (eq. (7)), 2) achievable per-cell capacity with FDMA and optimal multicell joint decoding (eq. (8) and (14)), 3) achievable per-cell capacity with TDMA and optimal multicell joint decoding (eq. (8) and (13)), 4) achievable per-cell capacity with FDMA and linear MMSE filtering (eq. (12) and (14)), 5) achievable per-cell capacity with TDMA and linear MMSE filtering (eq. (12) and (13)). The analytical curves are verified by running Monte Carlo simulations over 100 random realizations of the system and by averaging the produced results. More specifically, for

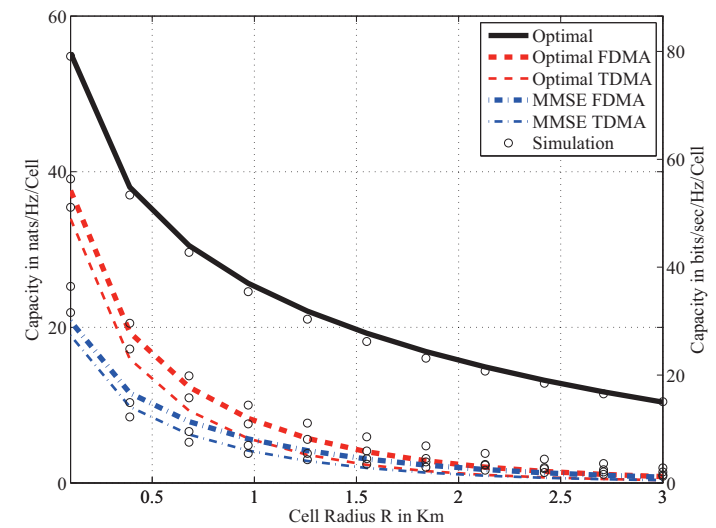

Figure 2: Per-cell capacity vs. cell radius $R$. Parameters: $K=20, n_{B S}=4, \mathrm{PAR}=2$.

each system realization the Gaussian complex matrix $\mathbf{G}$ is constructed by randomly generating Gaussian i.i.d. c.c.s. fading coefficients with unit variance. Similarly, the variance profile matrix $\boldsymbol{\Sigma}$ is constructed by randomly placing $K$ UTs according to a uniform distribution in the coverage range of each cell and by calculating the variance profile coefficients. After constructing the channel matrix $\mathbf{H}=\boldsymbol{\Sigma} \odot \mathbf{G}$, the optimal per-cell sum-rate capacity is calculated by [13]

$$
C_{\text {opt }}=\frac{1}{N} \mathbb{E}\left[\log \operatorname{det}\left(\mathbf{I}_{N n_{B S}}+\gamma \mathbf{H} \mathbf{H}^{\dagger}\right)\right] .
$$

In addition, the intra-cell orthogonalization channel matrix $\mathbf{H}_{\mathbf{o}}$ is constructed by randomly selecting $n_{B S}$ out of the $K$ UTs of each cell and concatenating the corresponding column vectors of $\mathbf{H}$. The achievable per-cell sum-rate with FDMA/TDMA and optimal multicell decoding is given by

$$
C_{\mathrm{opt}}=\frac{1}{N} \mathbb{E}\left[\log \operatorname{det}\left(\mathbf{I}_{N n_{B S}}+\gamma \mathbf{H}_{\mathbf{o}} \mathbf{H}_{\mathbf{o}}^{\dagger}\right)\right],
$$

where $\gamma=\gamma_{\mathrm{F}}$ for FDMA and $\gamma=\gamma_{\mathrm{T}}$ for TDMA. Similarly, the achievable per-cell sum-rate with FDMA/TDMA and linear MMSE filtering is given by

$$
C_{\mathrm{mmse}}=-\frac{1}{N} \mathbb{E}\left[\sum_{k=1}^{N n_{B S}} \log \left[\left(\mathbf{I}_{N n_{B S}}+\gamma \mathbf{H}_{\mathbf{o}}^{\dagger} \mathbf{H}_{\mathbf{o}}\right)^{-1}\right]_{k, k}\right] .
$$

\subsection{Achievable Sum-rate Results}

Figure 2 depicts the per-cell capacity versus the cell radius $R$ for the five considered scenarios, while preserving a fixed number of UTs per cell $K$. It should be noted that continuous lines represent analytical plots, whereas circle points represent simulation values. As Figure 2 illustrates, there is a close agreement between analysis and simulation for the considered range of values. Moreover, Figure 3 illustrates the per-cell capacity for fixed cell radius and number of BS antennas, while the number of UTs per cell increases. It is shown that FDMA sum-rate increases while TDMA sumrate remains fixed with the number of UTs per cell due to the peak power saturation. What is more, the complexity of the multicell receiver does not increase with the number of UTs per cell, since only $N n_{B S}$ out of $K N$ are processed per channel use for the sub-optimal schemes. Finally, Figure 4 depicts the per-cell capacity for fixed cell radius and UTs 


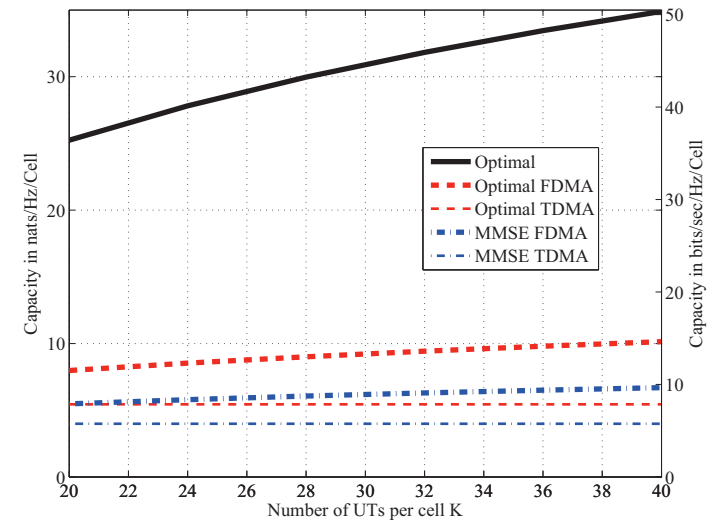

Figure 3: Per-cell capacity vs. number of UTs per cell $K$. Parameters: $R=1 K m, n_{B S}=4, \operatorname{PAR}=2$.

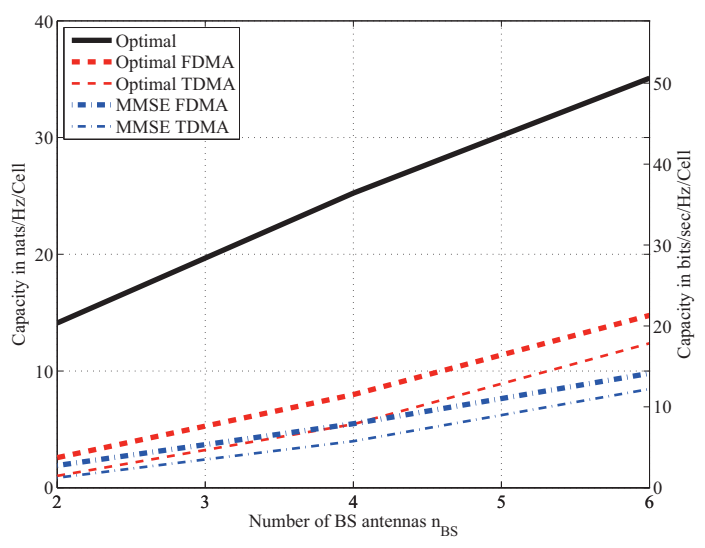

Figure 4: Per-cell capacity vs. number of BS antennas $n_{B S}$. Parameters: $R=1 K m, K=20, \mathrm{PAR}=2$.

per cell, while the number of BS antennas increases. As it can be seen, even with the suboptimal schemes an almost linear scaling of the achieved sum-rate with the number of BS antennas is observed.

\section{CONCLUSION}

In the context of multicell-decoding systems, the complexity of the optimum multicell joint decoder scales with the number of system UTs and becomes impractical even for moderately sized cellular systems. In this paper, we have investigated multiple-access techniques and multiuser receivers, which can reduce the system complexity. In this direction, we considered intra-cell orthogonalization as a means of decoupling the multiuser receiver's load from the number of UTs. In addition, we investigated the performance of the suboptimal but efficient linear MMSE filtering, followed by single-user decoding. The main conclusion of this study is that linear MMSE filtering combined with multiple BS antennas and intra-cell orthogonalization can provide a considerable capacity enhancement for an affordable complexity level. From a system-design point of view, a capacity versus complexity trade-off can be achieved by varying the number of $\mathrm{BS}$ antennas and as a result the size of the UT group which has to be jointly filtered. Finally,
FDMA is more efficient than TDMA as an intra-cell orthogonalization technique, when the UT transmissions are peakpower constrained.

\section{Acknowledgment}

The work reported in this paper has formed part of the "Fundamental Limits to Wireless Network Capacity" Elective Research Programme of the Virtual Centre of Excellence in Mobile \& Personal Communications, Mobile VCE, www.mobilevce.com. This research has been funded by the following Industrial Companies who are Members of Mobile VCE - BBC, BT, Huawei, Nokia, Nokia Siemens Networks, Nortel, Vodafone.

\section{REFERENCES}

[1] A.D. Wyner. Shannon-theoretic approach to a Gaussian cellular multiple-access channel. IEEE Trans. Inform. Theory, 40(6):1713-1727, Nov 1994.

[2] O. Somekh and S. Shamai. Shannon-theoretic approach to a Gaussian cellular multiple-access channel with fading. IEEE Trans. Inform. Theory, 46(4):1401-1425, Jul 2000.

[3] N.A. Letzepis. Gaussian Cellular Muptiple Access Channels. PhD thesis, Institute for Telecommunications Research, University of South Australia, Dec 2005.

[4] Huaiyu Dai and H.V. Poor. Asymptotic spectral efficiency of multicell MIMO systems with frequency-flat fading. IEEE Trans. Signal Processing, 51(11):2976-2988, Nov 2003.

[5] S. Chatzinotas, M.A. Imran, and C. Tzaras. On the capacity of variable density cellular systems under multicell decoding. IEEE Commun. Lett., 12(7):496 498, Jul 2008.

[6] O. Somekh, B.M. Zaidel, and S. Shamai. Sum rate characterization of joint multiple cell-site processing. IEEE Trans. Inform. Theory, 53(12):4473-4497, Dec. 2007.

[7] D. Aktas, M.N. Bacha, J.S. Evans, and S.V. Hanly. Scaling results on the sum capacity of cellular networks with MIMO links. IEEE Trans. Inform. Theory, 52(7):3264-3274, July 2006.

[8] S. Verdu. Multiuser Detection. Cambridge University Press, UK, 1998.

[9] Antonio M. Tulino and Sergio Verdu. Random matrix theory and wireless communications. Commun. Inf. Theory, 1(1):1-182, 2004.

[10] U. Madhow and M.L. Honig. MMSE interference suppression for direct-sequence spread-spectrum CDMA. IEEE Trans. Commun., 42(12):3178-3188, Dec 1994.

[11] D.N.C. Tse and S.V. Hanly. Linear multiuser receivers: effective interference, effective bandwidth and user capacity. IEEE Trans. Inform. Theory, 45(2):641-657, Mar 1999.

[12] ETSI TR 125996 V7.0.0. UMTS spatial channel model for MIMO simulations (3GPP TR 25.996 ver. 7.0.0 rel. 7), Jun 2007.

[13] I. E. Telatar. Capacity of multi-antenna Gaussian channels. European Transactions on Telecommunications, 10(6):585-595, Nov 1999. 